\title{
Sharpness of Wilker and Huygens type inequalities
}

\author{
Chao-Ping Chen $^{1 *}$ and Wing-Sum Cheung ${ }^{2}$
}

\author{
* Correspondence: \\ chenchaoping@sohu.com \\ ${ }^{1}$ School of Mathematics and \\ Informatics, Henan Polytechnic \\ University, Jiaozuo City 454003 \\ Henan Province, People's Republic \\ of China \\ Full list of author information is \\ available at the end of the article
}

\section{Abstract}

We present an elementary proof of Wilker's inequality involving trigonometric functions, and establish sharp Wilker and Huygens type inequalities.

Mathematics Subject Classification 2010: $26 \mathrm{D05}$.

Keywords: inequalities, trigonometric functions

\section{Introduction}

Wilker in [1] proposed two open problems:

(a) Prove that if $0<x<\pi / 2$, then

$$
\left(\frac{\sin x}{x}\right)^{2}+\frac{\tan x}{x}>2
$$

1

(b) Find the largest constant c such that

$$
\left(\frac{\sin x}{x}\right)^{2}+\frac{\tan x}{x}>2+c x^{3} \tan x
$$

for $0<x<\pi / 2$.

In [2], inequality (1) was proved, and the following inequality

$$
2+\left(\frac{2}{\pi}\right)^{4} x^{3} \tan x<\left(\frac{\sin x}{x}\right)^{2}+\frac{\tan x}{x}<2+\frac{8}{45} x^{3} \tan x \text { for } 0<x<\frac{\pi}{2}
$$

where the constants $\left(\frac{2}{\pi}\right)^{4}$ and $\frac{8}{45}$ are best possible, was also established.

Wilker type inequalities (1) and (2) have attracted much interest of many mathematicians and have motivated a large number of research papers involving different proofs and various generalizations and improvements (cf. [2-13] and the references cited therein). A brief survey of some old and new inequalities associated with trigonometric functions can be found in [14]. These include (among other results) Wilker's inequality.

\section{包 Springer}

(C) 2012 Chen and Cheung; licensee Springer. This is an Open Access article distributed under the terms of the Creative Commons Attribution License (http://creativecommons.org/licenses/by/2.0), which permits unrestricted use, distribution, and reproduction in any medium, provided the original work is properly cited. 
Another inequality which is of interest to us is Huygens [15] inequality, which asserts that

$$
2\left(\frac{\sin x}{x}\right)+\frac{\tan x}{x}>3 \text { for all } 0<|x|<\frac{\pi}{2} .
$$

Neuman and Sándor [16] have pointed out that (3) implies (1). In [17], Zhu established some new inequalities of the Huygens type for trigonometric and hyperbolic functions. Baricz and Sándor [18] pointed out that inequalities (1) and (3) are simple consequences of the arithmetic-geometric mean inequality together with the wellknown Lazarević-type inequality [[19], p. 238]

$$
(\cos x)^{1 / 3}<\frac{\sin x}{x} \text { for all } 0<|x|<\frac{\pi}{2},
$$

or equivalently,

$$
\left(\frac{\sin x}{x}\right)^{2} \frac{\tan x}{x}>1 \quad \text { for all } \quad 0<|x|<\frac{\pi}{2} .
$$

Wu and Srivastava [[7], Lemma 3] established another inequality

$$
\left(\frac{x}{\sin }\right)^{2}+\frac{x}{\tan x}>2 \text { for all } 0<|x|<\frac{\pi}{2} .
$$

In [20], Chen and Cheung showed that Wilker inequality (1), Huygens inequality (3), Lazarević-type inequality (4) and Wu-Srivastava inequality (5) can be grouped into the following inequality chain:

$$
\begin{aligned}
\frac{(\sin x / x)^{2}+\tan x / x}{2} & >\frac{2(\sin x / x)+\tan x / x}{3}>\sqrt[3]{\left(\frac{\sin x}{x}\right)^{2} \frac{\tan x}{x}}>1 \\
& >\frac{2}{1 /(\sin x / x)^{2}+1 /(\tan x / x)}, \quad 0<|x|<\frac{\pi}{2}
\end{aligned}
$$

in terms of the arithmetic, geometric and harmonic means.

In this article, we present an elementary proof of Wilker's inequality (2), and we establish sharp Wilker and Huygens type inequalities.

The following elementary power series expansions are useful in our investigation.

$$
\begin{array}{ll}
\sin x=\sum_{n=0}^{\infty}(-1)^{n} \frac{x^{2 n+1}}{(2 n+1) !}, & |x|<\infty, \\
\cos x=\sum_{n=0}^{\infty}(-1)^{n} \frac{x^{2 n}}{(2 n) !}, & |x|<\infty, \\
\tan x=\sum_{n=1}^{\infty} \frac{2^{2 n}\left(2^{2 n}-1\right)(-1)^{n-1} B_{2 n}}{(2 n) !} x^{2 n-1}, & |x|<\frac{\pi}{2}, \\
x \cot x=\sum_{n=0}^{\infty}(-1)^{n} B_{2 n} \frac{(2 x)^{2 n}}{(2 n) !}, & 0<|x|<\pi .
\end{array}
$$


where $B_{n}(n=0,1,2, \ldots)$ are Bernoulli numbers, defined by

$$
\frac{t}{e^{t}-1}=\sum_{n=0}^{\infty} B_{n} \frac{t^{n}}{n !}
$$

The following lemma is also needed in the sequel.

Lemma 1. [21] Let $a_{n} \in \mathbb{R}$ and $b_{n}>0, n=0,1,2, \ldots$ be real numbers with $\left\{\frac{a_{n}}{b_{n}}\right\}_{n=1}^{\infty}$ being strictly increasing (respectively, decreasing). If the power series $B(x):=\sum_{n=0}^{\infty} b_{n} x^{n}$ and $B(x):=\sum_{n=0}^{\infty} b_{n} x^{n}$ are convergent for $|x|<R$, then the function $A$ $(x) / B(x)$ is strictly increasing (respectively, decreasing) on $(0, R)$.

\section{An elementary proof of Wilker's inequality (2)}

Proof of (2). Consider the function

$$
f(x):=\frac{\left(\frac{\sin x}{x}\right)^{2}+\frac{\tan x}{x}-2}{x^{3} \tan x}, \quad 0<x<\frac{\pi}{2} .
$$

By using power series expansions (7) and (8), we obtain

$$
\begin{aligned}
-x^{6} \sin ^{2} x f^{\prime}(x) & =-3 x^{2} \sin (2 x)+5 \sin ^{3} x \cos x+5 x-2 x^{3}+2 x \cos ^{4} x-7 x \cos ^{2} x \\
& =\left(-3 x^{2}+\frac{5}{4}\right) \sin (2 x)-\frac{5}{8} \sin (4 x)-2 x^{3}+\frac{9}{4} x+\frac{1}{4} x \cos (4 x)-\frac{5}{2} x \cos (2 x) \\
& =\sum_{n=4}^{\infty}(-1)^{n} u_{n}(x) \\
& =\frac{16}{945} x^{9}-\frac{16}{1575} x^{11}+\frac{16}{7425} x^{13}-\frac{11072}{42567525} x^{15}+\sum_{n=8}^{\infty}(-1)^{n} u_{n}(x)
\end{aligned}
$$

where

$$
u_{n}(x):=\frac{\left((2 n-9) 4^{n-1}+6 n^{2}-2 n\right) 4^{n}}{(2 n+1) !} x^{2 n+1}
$$

Elementary calculations reveal that, for $0<x<\pi / 2$ and $n \geq 8$,

$$
\begin{aligned}
\frac{u_{n+1}(x)}{u_{n}(x)} & =8 x^{2} \frac{6 n^{2}+10 n+4+(2 n-7) 4^{n}}{(n+1)(2 n+3)\left(24 n^{2}-8 n+(2 n-9) 4^{n}\right)} \\
& <8\left(\frac{\pi}{2}\right)^{2} \frac{6 n^{2}+10 n+4+(2 n-7) 4^{n}}{(n+1)(2 n+3)\left(24 n^{2}-8 n+(2 n-9) 4^{n}\right)} \\
& =\frac{\pi^{2}}{2 n+3} \frac{12 n^{2}+20 n+8+2(2 n-7) 4^{n}}{(n+1)\left(24 n^{2}-8 n+(2 n-9) 4^{n}\right)} .
\end{aligned}
$$

Write

$$
\alpha_{n}:=\frac{\pi^{2}}{2 n+3} \frac{12 n^{2}+20 n+8+2(2 n-7) 4^{n}}{(n+1)\left(24 n^{2}-8 n+(2 n-9) 4^{n}\right)} .
$$

It is easy to see that for $n \geq 8$,

$$
\frac{12 n^{2}+20 n+8+2(2 n-7) 4^{n}}{(n+1)\left(24 n^{2}-8 n+(2 n-9) 4^{n}\right)}<1 .
$$


Hence for all $0<x<\pi / 2$ and $n \geq 8$,

$$
\frac{u_{n+1}(x)}{u_{n}(x)}<\alpha_{n}<\frac{\pi^{2}}{2 n+3}<1 .
$$

Therefore, for fixed $x \in(0, \pi / 2)$, the sequence $n \mapsto u_{n}(x)$ is strictly decreasing with regard to $n \geq 8$. Hence, for $0<x<\pi / 2$,

$$
\begin{aligned}
-x^{6} \sin ^{2} x f^{\prime}(x) & >\frac{16}{945} x^{9}-\frac{16}{1575} x^{11}+\frac{16}{7425} x^{13}-\frac{11072}{42567525} x^{15} \\
& =x^{9}\left(\frac{16}{945}-\frac{16}{1575} x^{2}+\frac{16}{7425} x^{4}-\frac{11072}{42567525} x^{6}\right) \\
& >0 .
\end{aligned}
$$

Hence $f(x)$ is strictly decreasing on $(0, \pi / 2)$. Noting that $\lim _{t \rightarrow 0^{+}} f(t)=\frac{8}{45}$, we have

$$
\frac{16}{\pi^{4}}=\lim _{t \rightarrow(\pi / 2)^{-}} f(t)<f(x)=\frac{\left(\frac{\sin x}{x}\right)^{2}+\frac{\tan x}{x}-2}{x^{3} \tan x}<\lim _{t \rightarrow 0^{+}} f(t)=\frac{8}{45}
$$

for all $x \in\left(0, \frac{\pi}{2}\right)$, with the constants $\frac{16}{\pi^{4}}$ and $\frac{8}{45}$ being best possible. This completes the proof of (2).

\section{Sharp Wilker's inequality}

By using power series expansions (8) and (9), we have, for $0<x<\pi / 2$,

$$
\begin{aligned}
\left(\frac{\sin x}{x}\right)^{2}+\frac{\tan x}{x} & =\frac{1}{2 x^{2}}=-\frac{1}{2 x^{2}} \cos (2 x)+\frac{\tan x}{x} \\
& =2+\sum_{k=3}^{\infty} \frac{\left(2\left(2^{2 k}-1\right)\left|B_{2 k}\right|-(-1)^{k}\right) 2^{2 k-1}}{(2 k) !} x^{2 k-2} .
\end{aligned}
$$

It is well known [[22], p. 805] that

$$
\frac{2(2 k) !}{(2 \pi)^{2 k}}<\left|B_{2 k}\right|<\frac{2(2 k) !}{(2 \pi)^{2 k}\left(1-2^{1-2 k}\right)}, \quad k \geq 1 .
$$

By (11), we find that

$$
2\left(2^{2 k}-1\right)\left|B_{2 k}\right|>2\left(2^{2 k}-1\right) \frac{2(2 k) !}{(2 \pi)^{2 k}}>1, \quad k \geq 3 .
$$

Hence, we have

$$
\begin{aligned}
\left(\frac{\sin x}{x}\right)^{2}+\frac{\tan x}{x}> & 2+\sum_{k=3}^{n} \frac{\left(2\left(2^{2 k}-1\right)\left|B_{2 k}\right|-(-1)^{k}\right) 2^{2 k-1}}{(2 k) !} x^{2 k-2} \\
= & 2+\frac{8}{45} x^{4}+\frac{16}{315} x^{6}+\frac{104}{4725} x^{8}+\frac{592}{66825} x^{10} \\
& +\cdots+\frac{\left(2\left(2^{2 n}-1\right)\left|B_{2 n}\right|-(-1)^{n}\right) 2^{2 n-1}}{(2 n) !} x^{2 n-2} .
\end{aligned}
$$

Motivated by (12), we are now in a position to establish our first main result. 
Theorem 1. (i) For $0<x<\pi / 2$, we have

$$
2+\frac{8}{45} x^{4}+\frac{16}{315} x^{5} \tan x<\left(\frac{\sin x}{x}\right)^{2}+\frac{\tan x}{x}<2+\frac{8}{45} x^{4}+\left(\frac{2}{\pi}\right)^{6} x^{5} \tan x .
$$

The constants $\frac{16}{315}$ and $\left(\frac{2}{\pi}\right)^{6}$ are best possible.

(ii) For $0<x<\pi / 2$, we have

$$
\begin{aligned}
2+\frac{8}{45} x^{4}+\frac{16}{315} x^{6}+\frac{104}{4725} x^{7} \tan x & <\left(\frac{\sin x}{x}\right)^{2}+\frac{\tan x}{x} \\
& <2+\frac{8}{45} x^{4}+\frac{16}{315} x^{6}+\left(\frac{2}{\pi}\right)^{8} x^{7} \tan x
\end{aligned}
$$

The constants $\frac{104}{4725}$ and $\left(\frac{2}{\pi}\right)^{8}$ are best possible.

Proof. We only prove inequality (13). The proof of (14) is analogous.

Consider the function

$$
\begin{aligned}
g(x): & =\frac{\left(\frac{\sin x}{x}\right)^{2}+\frac{\tan x}{x}-2-\frac{8}{45} x^{4}}{x^{5} \tan x} \\
& =\frac{\sin (2 x)}{2 x^{7}}+\frac{1}{x^{6}}-\frac{2 \cot x}{x^{5}}-\frac{8 \cot x}{45 x}, \quad 0<x<\frac{\pi}{2} .
\end{aligned}
$$

By using power series expansions (7) and (10), we find that

$$
g(x)=\sum_{n=3}^{\infty} \beta_{n} 2^{2 n-1} x^{2 n-6}
$$

where

$$
\beta_{n}:=\frac{4 \cdot\left|B_{2 n}\right|}{(2 n) !}+\frac{\left|B_{2(n-2)}\right|}{45 \cdot(2 n-4) !}+(-1)^{n} \frac{2}{(2 n+1) !}, \quad n \geq 3 .
$$

By (11), we obtain

$$
\begin{aligned}
\frac{4 \cdot\left|B_{2 n}\right|}{(2 n) !}+\frac{\left|B_{2(n-2)}\right|}{45 \cdot(2 n-4) !} & >\frac{4}{(2 n) !} \frac{2(2 n) !}{(2 \pi)^{2 n}}+\frac{1}{45 \cdot(2 n-4) !} \frac{2(2 n-4) !}{(2 \pi)^{2 n-4}} \\
& =\frac{180+2 \cdot(2 \pi)^{4}}{45 \cdot(2 \pi)^{2 n}} .
\end{aligned}
$$

By induction on $n$, it is easy to see that

$$
\frac{180+2 \cdot(2 \pi)^{4}}{45 \cdot(2 \pi)^{2 n}}>\frac{2}{(2 n+1) !} \quad \text { for all } n \geq 3
$$


Hence $\beta_{n}>0$ for $n \geq 3$, and we have

$$
g^{\prime}(x)=\sum_{n=4}^{\infty} b_{n} 2^{2 n-1}(2 n-6) x^{2 n-7}>0, \quad 0<x<\frac{\pi}{2} .
$$

Therefore, $g(x)$ is strictly increasing on $(0, \pi / 2)$. Noting that $\lim _{t \rightarrow(\pi / 2)^{-}} g(t)=\left(\frac{2}{\pi}\right)^{6}$, we have

$$
\frac{16}{315}=\lim _{t \rightarrow 0^{+}} g(t)<g(x)=\frac{\left(\frac{\sin x}{x}\right)^{2}+\frac{\tan x}{x}-2-\frac{8}{45} x^{4}}{x^{5} \tan x}<\lim _{t \rightarrow(\pi / 2)^{-}} g(t)=\left(\frac{2}{\pi}\right)^{6}
$$

for all $x \in\left(0, \frac{\pi}{2}\right)$, with the constants $\frac{16}{315}$ and $\left(\frac{2}{\pi}\right)^{6}$ being best possible. This completes the proof of (13).

Remark 1. Inequality (14) is sharper than (13). On the other hand, there is no strict comparison between inequalities (2) and (13). There is no strict comparison between inequalities (2) and (14) either.

In view of inequalities (13) and (14), we propose the following conjecture.

Conjecture 1. For $0<x<\pi / 2$ and $n \geq 3$, we have

$$
\begin{aligned}
& +\sum_{k=3}^{n} \frac{\left(2\left(2^{2 k}-1\right)\left|B_{2 k}\right|-(-1)^{k}\right) 2^{2 k-1}}{(2 k) !} x^{2 k-2} \\
& +\frac{\left(2\left(2^{2 n+2}-1\right)\left|B_{2 n+2}\right|-(-1)^{n+1}\right) 2^{2 n+1}}{(2 n+2) !} x^{2 n-1} \tan x \\
< & \left(\frac{\sin x}{x}\right)^{2}+\frac{\tan x}{x} \\
< & 2+\sum_{k=3}^{n} \frac{\left(2\left(2^{2 k}-1\right)\left|B_{2 k}\right|-(-1)^{k}\right) 2^{2 k-1}}{(2 k) !} x^{2 k-2}+\left(\frac{2}{\pi}\right)^{2 n} x^{2 n-1} \tan x .
\end{aligned}
$$

\section{Sharp the Wu-Srivastava inequality}

By using power series expansion (10), we obtain for $0<x<\pi / 2$,

$$
\csc ^{2} x=-(\cot x)^{\prime}=\frac{1}{x^{2}}+\sum_{k=1}^{\infty} \frac{2^{2 k}\left(2 k-1\left|B_{2 k}\right|\right)}{(2 k) !} x^{2 k-2} .
$$

Hence for $0<x<\pi / 2$,

$$
\begin{aligned}
\left(\frac{x}{\sin x}\right)^{2}+\frac{x}{\tan x} & =x^{2} \csc ^{2} x+x \cot x \\
& =2+\sum_{k=2}^{\infty} \frac{2^{2 k+1}(k-1)\left|B_{2 k}\right|}{(2 k) !} x^{2 k} \\
& =2+\frac{2}{45} x^{4}+\frac{8}{945} x^{6}+\frac{2}{1575} x^{8}+\frac{16}{93555} x^{10}+\cdots .
\end{aligned}
$$

Motivated by (16), we establish our second main result: 
Theorem 2. (i) For $0<x<\pi / 2$, we have

$$
\left(\frac{x}{\sin x}\right)^{2}+\frac{x}{\tan x}<2+\frac{2}{45} x^{3} \tan x
$$

The constant $\frac{2}{45}$ is best possible.

(ii) For $0<x<\pi / 2$, we have

$$
\left(\frac{x}{\sin x}\right)^{2}+\frac{x}{\tan x}<2+\frac{2}{45} x^{4}+\frac{8}{945} x^{5} \tan x .
$$

The constant $\frac{8}{945}$ is best possible.

Proof. We only prove inequality (18). The proof of (17) is analogous.

Consider the function

$$
G(x):=\frac{\left(\frac{x}{\sin x}\right)^{2}+\frac{x}{\tan x}-2-\frac{2}{45} x^{4}}{x^{5} \tan x}=\frac{A(x)}{B(x)}
$$

where

$$
\begin{aligned}
A(x): & =\left(\frac{x}{\sin x}\right)^{2}+\frac{x}{\tan x}-2-\frac{2}{45} x^{4} \\
& =\sum_{n=1}^{\infty} \frac{2^{2 n+5}(n+1)\left|B_{2(n+2)}\right|}{(2 n+4) !} x^{2 n+4}=\sum_{n=1}^{\infty} a_{n} x^{2 n+4}
\end{aligned}
$$

with

$$
a_{n}:=\frac{2^{2 n+5}(n+1)\left|B_{2(n+2)}\right|}{(2 n+4) !}
$$

and

$$
B(x):=x^{5} \tan x=\sum_{n=1}^{\infty} \frac{2^{2 n}\left(2^{2 n}-1\right)\left|B_{2 n}\right|}{(2 n) !} x^{2 n+4}=\sum_{n=1}^{\infty} b_{n} x^{2 n+4}
$$

with

$$
b_{n}:=\frac{2^{2 n}\left(2^{2 n}-1\right)\left|B_{2 n}\right|}{(2 n) !}
$$

We claim that the function $G(x)$ is strictly decreasing on $(0, \pi / 2)$. By Lemma 1 , it suffices to show that

$$
\frac{a_{n}}{b_{n}}>\frac{a_{n+1}}{b_{n+1}}, \quad n \geq 1
$$

It is known [[23], p. 96] that

$$
\frac{2 \cdot(2 n) !}{(2 \pi)^{2 n}}<\left|B_{2 n}\right|<\frac{\pi^{2}(2 n) !}{3(2 \pi)^{2 n}}, \quad n \geq 1 .
$$


By using (20), we obtain

$$
\frac{a_{n}}{b_{n}}=\frac{2^{5}(n+1)\left|B_{2(n+2)}\right|}{(2 n+4) !} \cdot \frac{(2 n) !}{\left(2^{2 n}-1\right)\left|B_{2 n}\right|}>\frac{192(n+1)}{\pi^{2}(2 \pi)^{4}\left(4^{n}-1\right)}
$$

and

$$
\frac{a_{n+1}}{b_{n+1}}=\frac{2^{5}(n+2)\left|B_{2(n+3)}\right|}{(2 n+6) !} \cdot \frac{(2 n+2) !}{\left(2^{2 n+2}-1\right)\left|B_{2(n+1)}\right|}<\frac{16 \pi^{2}(n+2)}{3(2 \pi)^{4}\left(4^{n+1}-1\right)} .
$$

So (19) is a consequence of the elementary inequality

$$
\frac{192(n+1)}{\pi^{2}(2 \pi)^{4}\left(4^{n}-1\right)}>\frac{16 \pi^{2}(n+2)}{3(2 \pi)^{4}\left(4^{n+1}-1\right)}
$$

which is equivalent to

$$
\frac{36(n+1)}{4^{n}-1}>\frac{\pi^{4}(n+2)}{4^{n+1}-1}, \quad n \geq 1 .
$$

The proof of the inequality (21) is not difficult, and is left with the readers. This proves the claim.

Noting that $\lim _{t \rightarrow 0^{+}} G(t)=\frac{8}{945}$, we have

$$
G(x)<\lim _{t \rightarrow 0^{+}} G(t)=\frac{8}{945} \quad \text { for all } x \in\left(0, \frac{\pi}{2}\right)
$$

with the constant $\frac{8}{945}$ being best possible. This completes the proof of (18).

In view of inequalities (17) and (18), we propose the following conjecture.

Conjecture 2. For $0<x<\pi / 2$ and $n \geq 1$,

$$
\left(\frac{x}{\sin x}\right)^{2}+\frac{x}{\tan x}<2+\sum_{k=2}^{n} \frac{(k-1) \cdot 2^{2 k+1}\left|B_{2 k}\right|}{(2 k) !} x^{2 k}+\frac{n \cdot 2^{2 n+3}\left|B_{2(n+1)}\right|}{(2 n+2) !} x^{2 n+1} \tan x .
$$

\section{Skarp Huygens inequality}

By using power series expansions (7) and (9), for $0<x<\pi / 2$, we have

$$
2\left(\frac{\sin x}{x}\right)+\frac{\tan x}{x}=3+\sum_{k=3}^{\infty}\left(\frac{2^{2 k}\left(2^{2 k}-1\right)\left|B_{2 k}\right|}{4 k}-(-1)^{k}\right) \frac{2 x^{2 k-2}}{(2 k-1) !} .
$$

By (11), we find that

$$
\frac{2^{2 k}\left(2^{2 k}-1\right)\left|B_{2 k}\right|}{4 k}>\frac{2^{2 k}\left(2^{2 k}-1\right)}{4 k} \frac{2(2 k) !}{(2 \pi)^{2 k}}=\frac{\left(2^{2 k}-1\right) \cdot(2 k-1) !}{\pi^{2 k}}>1, \quad k \geq 3 .
$$


Hence we have

$$
\begin{aligned}
2\left(\frac{\sin x}{x}\right)+\frac{\tan x}{x}> & 3+\sum_{k=3}^{n}\left(\frac{2^{2 k}\left(2^{2 k}-1\right)\left|B_{2 k}\right|}{4 k}-(-1)^{k}\right) \frac{2 x^{2 k-2}}{(2 k-1) !} \\
= & 3+\frac{3}{20} x^{4}+\frac{3}{56} x^{6}+\frac{7}{320} x^{8}+\frac{3931}{443520} x^{10} \\
& +\cdots+\left(\frac{\left(2^{2 n}\left(2^{2 n}-1\right)\left|B_{2 n}\right|\right)}{4 n}-(-1)^{n}\right) \frac{2 x^{2 n-2}}{(2 n-1) !} .
\end{aligned}
$$

Motivated by (23), we establish our third main result:

Theorem 3. (i) For $0<x<\pi / 2$, we have

$$
3+\frac{3}{20} x^{3} \tan x<2\left(\frac{\sin x}{x}\right)+\frac{\tan x}{x}<3+\left(\frac{2}{\pi}\right)^{4} x^{3} \tan x .
$$

The constants $\frac{3}{20}$ and $\left(\frac{2}{\pi}\right)^{4}$ are best possible.

(ii) For $0<x<\pi / 2$, we have

$$
3+\frac{3}{20} x^{4}+\frac{3}{56} x^{5} \tan x<2\left(\frac{\sin x}{x}\right)+\frac{\tan x}{x}<3+\frac{3}{20} x^{4}+\left(\frac{2}{\pi}\right)^{6} x^{5} \tan x .
$$

The constants $\frac{3}{56}$ and $\left(\frac{2}{\pi}\right)^{6}$ are best possible.

Proof. We only prove inequality (25). The proof of (24) is analogous.

Consider the function

$$
\begin{aligned}
h(x): & =\frac{2\left(\frac{\sin x}{x}\right)+\frac{\tan x}{x}-3-\frac{3}{20} x^{4}}{x^{5} \tan x} \\
& =\frac{2 \cos x}{x^{6}}+\frac{1}{x^{6}}-\frac{3 \cot x}{x^{5}}-\frac{3 \cot x}{20 x}, \quad 0<x<\frac{\pi}{2} .
\end{aligned}
$$

By using power series expansions (8) and (10), we find that

$$
h(x)=\sum_{n=3}^{\infty} c_{n} x^{2 n-6}
$$

where

$$
c_{n}:=\frac{3 \cdot 2^{2 n}\left|B_{2 n}\right|}{(2 n) !}+\frac{3 \cdot 2^{2 n-4}\left|B_{2(n-2)}\right|}{20 \cdot(2 n-4) !}+(-1)^{n} \frac{2}{(2 n) !}, \quad n \geq 3 .
$$

By (11), we obtain

$$
\begin{aligned}
\frac{3 \cdot 2^{2 n}\left|B_{2 n}\right|}{(2 n) !}+\frac{3 \cdot 2^{2 n-4}\left|B_{2(n-2)}\right|}{20 \cdot(2 n-4) !} & >\frac{3 \cdot 2^{2 n}}{(2 n) !} \frac{2(2 n) !}{(2 \pi)^{2 n}}+\frac{3 \cdot 2^{2 n-4}}{20 \cdot(2 n-4) !} \frac{2(2 n-4) !}{(2 \pi)^{2 n-4}} \\
& =\frac{60+3 \cdot \pi^{4}}{10 \cdot \pi^{2 n}} .
\end{aligned}
$$


By induction on $n$, it is easy to show that

$$
\frac{60+3 \cdot \pi^{4}}{10 \cdot \pi^{2 n}}>\frac{2}{(2 n) !} \text { for all } n \geq 3 .
$$

Hence $c_{n}>0$ for $n \geq 3$, and we have

$$
h^{\prime}(x)=\sum_{n=4}^{\infty} c_{n}(2 n-6) x^{2 n-7}>0 \quad \text { for all } 0<x<\frac{\pi}{2} .
$$

Therefore, $h(x)$ is strictly increasing on $(0, \pi / 2)$. Noting that $\lim _{t \rightarrow 0^{+}} h(t)=\frac{3}{56}$ and $\lim _{t \rightarrow(\pi / 2)-} h(t)=\left(\frac{2}{\pi}\right)^{6}$, we have

$$
\frac{3}{56}=\lim _{t \rightarrow 0^{+}} h(t)<h(x)=\frac{2\left(\frac{\sin x}{x}\right)+\frac{\tan x}{x}-3-\frac{3}{20} x^{4}}{x^{5} \tan x}<\lim _{t \rightarrow(\pi / 2)^{-}} h(t)=\left(\frac{2}{\pi}\right)^{6}
$$

for all $x \in\left(0, \frac{\pi}{2}\right)$ with the constants $\frac{3}{56}$ and $\left(\frac{2}{\pi}\right)^{6}$ being possible. This completes the proof of (25).

Remark 2. There is no strict comparison between inequalities (24) and (25).

In view of inequalities (24) and (25), we propose the following conjecture.

Conjecture 3. For $0<x<\pi / 2$ and $n \geq 2$, we have

$$
\begin{aligned}
& 3+\sum_{k=3}^{n}\left(\frac{2^{2 k}\left(2^{2 k}-1\right)\left|B_{2 k}\right|}{4 k}-(-1)^{k}\right) \frac{2}{(2 k-1) !} x^{2 k-2} \\
& +\left(\frac{2^{2 n+2}\left(2^{2 n+2}-1\right)\left|B_{2 n+2}\right|}{4(n+1)}-(-1)^{n+1}\right) \frac{2}{(2 n+1) !} x^{2 n-1} \tan x \\
< & 2\left(\frac{\sin x}{x}\right)+\frac{\tan x}{x} \\
< & 3+\sum_{k=3}^{n}\left(\frac{2^{2 k}\left(2^{2 k}-1\right)\left|B_{2 k}\right|}{4 k}-(-1)^{k}\right) \frac{2}{(2 k-1) !} x^{2 k-2}+\left(\frac{2}{\pi}\right)^{2 n} x^{2 n-1} \tan x .
\end{aligned}
$$

\section{Acknowledgements}

The research is supported in part by the Research Grants Council of the Hong Kong SAR, Project No. HKU7016/07P

\section{Author details}

ISchool of Mathematics and Informatics, Henan Polytechnic University, Jiaozuo City 454003, Henan Province, People's

Republic of China ${ }^{2}$ Department of Mathematics, The University of Hong Kong, Pokfulam Road, Hong Kong, China

\section{Authors' contributions}

All authors read and approved the final manuscript.

\section{Competing interests}

The authors declare that they have no competing interests.

Received: 29 June 2011 Accepted: 28 March 2012 Published: 28 March 2012

\section{References}

1. Wilker, JB: Problem E 3306. Am Math Mon. 96,55 (1989). doi:10.2307/2323260

2. Sumner, JS, Jagers, AA, Vowe, M, Anglesio, J: Inequalities involving trigonometric functions. Am Math Mon. 98, 264-267 (1991). doi:10.2307/2325035 
3. Guo, BN, Qiao, BM, Qi, F, Li, W: On new proofs of Wilker inequalities involving trigonometric functions. Math Inequal Appl. 6, 19-22 (2003)

4. Mortitc, C: The natural approach of Wilker-Cusa-Huygens inequalities. Math Inequal Appl. 14, 535-541 (2011)

5. Neuman, E: On Wilker and Huygens type inequalities. Math Inequal Appl. (in press)

6. Pinelis, I: L'Hospital rules of monotonicity and Wilker-Anglesio inequality. Am Math Mon. 111, 905-909 (2004). doi:10.2307/4145099

7. $\mathrm{Wu}, \mathrm{SH}$, Srivastava, HM: A weighted and exponential generalization of Wilker's inequality and its applications. Integr Trans Spec Funct. 18, 529-535 (2007). doi:10.1080/10652460701284164

8. Wu, SH, Srivastava, HM: A further refinement of Wilker's inequality. Integr Trans Spec Funct. 19, 757-765 (2008). doi:10.1080/10652460802340931

9. Zhang, L, Zhu, L: A new elementary proof of Wilker's inequalities. Math Inequal Appl. 11, 149-151 (2008)

10. Zhu, L: A new simple proof of Wilker's inequality. Math Inequal Appl. 8, 749-750 (2005)

11. Zhu, L: On Wilker-type inequalities. Math Inequal Appl. 10, 727-731 (2007)

12. Zhu, L: Some new Wilker-type inequalities for circular and hyperbolic functions. Abstr Appl Anal 2009, 9 (2009). (Article ID 485842)

13. Zhu, L: A source of inequalities for circular functions. Comput Math Appl. 58, 1998-2004 (2009). doi:10.1016/j. camwa.2009.07.076

14. Srivastava, R: Some families of integral, trigonometric and other related inequalities. Appl Math Inf Sci. 5, 342-360 (2011)

15. Huygens, C: Oeuvres Completes 1888-1940. Société Hollondaise des Science, Haga

16. Neuman, E, Sándor, J: On some inequalities involving trigonometric and hyperbolic functions with emphasis on the Cusa-Huygens, Wilker, and Huygens inequalities. Math Inequal Appl. 13, 715-723 (2010)

17. Zhu, L: Some new inequalities of the Huygens type. Comput Math Appl. 58, 1180-1182 (2009). doi:10.1016/j. camwa.2009.07.045

18. Baricz, A, Sándor, J: Extensions of generalized Wilker inequality to Bessel functions. J Math Inequal. 2, 397-406 (2008)

19. Mitrinović, DS: Analytic Inequalities. Springer-Verlag, Berlin (1970)

20. Chen, CP, Cheung, WS: Wilker- and Huygens-type inequalities and solution to Oppenheim's problem. Integr Trans Spec Funct. (in press)

21. Ponnusamy, S, Vuorinen, M: Asymptotic expansions and inequalities for hypergeometric functions. Mathematika. 44, 278-301 (1997). doi:10.1112/S0025579300012602

22. Abramowitz, M, Stegun, IA: Handbook of Mathematical Functions with Formulas, Graphs, and Mathematical Tables. In Appl Math Ser National Bureau of Standards, vol. 55,Washington, D.C (1972)

23. Kuang, J-Ch: Applied Inequalities. Shandong Science and Technology Press, Jinan City, Shandong Province, China (Chinese), 3 (2004)

doi:10.1186/1029-242X-2012-72

Cite this article as: Chen and Cheung: Sharpness of Wilker and Huygens type inequalities. Journal of Inequalities and Applications 2012 2012:72.

\section{Submit your manuscript to a SpringerOpen ${ }^{\odot}$ journal and benefit from:}

- Convenient online submission

- Rigorous peer review

- Immediate publication on acceptance

- Open access: articles freely available online

- High visibility within the field

- Retaining the copyright to your article

Submit your next manuscript at $\gg$ springeropen.com 